\title{
A Longitudinal Genetic Study of Vocabulary Knowledge in Adults
}

\author{
Stéphanie M. van den Berg, Daniëlle Posthuma, and Dorret I. Boomsma \\ Department of Biological Psychology, Vrije Universiteit Amsterdam, the Netherlands
}

\begin{abstract}
/ocabulary test scores were obtained from a total of 997 adults, all twins or a sibling of twins in this study. Some $(N=217)$ individuals were tested twice, around 6 years apart. Heritability varied from $50 \%$ at the first test occasion to $63 \%$ at the second test occasion. The correlation of scores across time was .74. Structural equation modelling showed that stability in vocabulary knowledge over time can largely (around $76 \%$ ) be explained by genetic factors. Part of the non-shared environmental variance was stable over time also. Any influence from shared environmental factors could not be detected. Results were similar for the two sexes, except that males generally outperformed females. Results were also similar for two age cohorts, except that the older cohort generally outperformed the younger cohort.
\end{abstract}

Since the publication of Noam Chomsky's (1957) Syntactic Structures, the question whether linguistic skills are innate or learned has been hotly debated. Observing that language acquisition shows the same order and tempo of development across cultures and languages, Chomsky argued that humans have an innate skill to grasp syntactic structures. Although words and syntactic rules vary from language to language, there are linguistic elements that all languages have in common. Chomsky claimed that what languages have in common is directly linked to the way the mind and brain work. The innate skill we humans have enables us to acquire language in the first place and in addition learn new languages. Thus, we have an inborn ability to acquire language, but what language we understand and speak (or sign), including what words we know and use, is determined by the environment we grow up in.

Interestingly, it has been observed in several studies that what words people know and/or use can be traced back to individual differences in genetic make-up. Size of vocabulary is a heritable trait. In one twin study, the estimated genetic contribution to (expressive) vocabulary in very young children (19 months old, as assessed by parents) was 39\% and the influence of the shared environment $51 \%$ (Dionne et al., 2003). Testing adolescent twins, Bratko (1996) reported an estimate of $61 \%$ for the genetic contribution to variation in vocabulary (more specifically, knowledge of synonyms and antonyms) but no influence of shared environment (see also Pedersen et al., 1992; Rowe et al., 1999).

Of course, heritability of vocabulary does not mean that people are born with a lexicon in their head, but rather that the environment that people grow up in is not the sole determinant of what words people know and use. The link between genes and vocabulary is possibly mediated by intelligence. In fact, many intelligence quotient (IQ) tests use vocabulary or knowledge of synonyms as an indicator of general cognitive ability (e.g., WAIS-III, Multidimensional Aptitude Battery, Groninger Intelligentie Test). Research has shown that knowing the meaning of uncommon words is highly associated with (other) measures of intelligence, not only at the phenotypic level but also at the genotypic level. Tests that estimate the relative size of a person's vocabulary load onto a general factor, commonly termed $g$, that explains correlations between different kinds of cognitive ability such as verbal skills, visuo-spatial skills, memory, and processing speed. Such a correlational structure also exists at the genotypic level, see for example Luciano et al. (2003).

The heritability of vocabulary is interesting because unlike intelligence indicators, such as processing speed and memory performance, vocabulary seems highly dependent on exposure to environmental stimuli. The same can be said of other forms of crystallized intelligence (Cattell, 1987). Without the right environmental influences (e.g., books, lectures, newspapers, documentaries, social interaction), one will never acquire the meaning of words that are less common. The genetic component in word knowledge therefore seems to be the result of an interaction between general intelligence and the right kind of intellectual environment, which in turn could be the result of an active or reactive genotype-environment correlation (Plomin et al., 1977; Scarr \& McCartney,

Received 23 October, 2003; accepted 8 January, 2004.

Address for correspondence: Stéphanie M. van den Berg, Vrije Universiteit Amsterdam, Department of Biological Psychology, Van der Boechorststraat 1, 1081 BT Amsterdam, the Netherlands. Email:SM.van.den.Berg@psy.vu.nl 
1983). In The Netherlands, high IQ children generally go to intellectually more stimulating high schools than do low IQ children. Inborn intelligence thus partly determines the environment which in turn determines vocabulary knowledge. Alternatively, or additionally, the genetic background of vocabulary may be mediated by an inborn curiosity or openness to experience (Aitken Harris, in press; Ashton et al., 2000; Costa \& McCrae, 1988). It is even conceivable that curiosity and openness modulate the intelligence by environmental interaction.

Here we describe a study into the heritability of vocabulary. Vocabulary size was assessed in 997 adults who came from a total of 413 extended twin families. As monozygotic twins share $100 \%$ of their genes and dizygotic twins and ordinary siblings share on average only $50 \%$ of their genes, variability in vocabulary size can be decomposed into genetic variance and environmental variance. Environmental variance can be further decomposed into environmental variance that is shared by all members raised in one family and variance that is unique for each individual family member, which includes error of measurement (Neale \& Cardon, 1992).

The uniqueness of the present study is that part of the sample was tested on two occasions, around 6 years apart. Analysing data from multiple testing occasions has several advantages. First, it is then possible to assess the reliability of the test and the stability of vocabulary size over time. Moreover, it allows determining whether the observed stability is due to genetic factors, environmental factors, or both. Second, studying the covariance over time eliminates the variance from measurement error as part of the unique environmental component. Third, it allows a more precise interpretation of the shared environment component. It is unclear what it means when a shared environment component is found at one test occasion. Such a component may mean either that there are important factors such as socioeconomic status of parents or diet during childhood, but it may also reflect the fact that family members are usually tested on the same day combined with the fact that a study usually takes a long time to complete. A shared environment component found with one test occasion therefore may only reflect seasonal effects, or that some words used in the test occurred more often in newspapers and television shows in the days or weeks preceding the day of the test (e.g., referendum, impeachment). As one does not expect these timerelated effects on members of the same family to correlate over a period of several years, one can expect the influence of one common shared environmental factor on the correlation of measures over time to diminish. The same reasoning applies to the unique environmental correlations over time, thereby giving way to the relative importance of a genetic factor operating across time. One may therefore a priori expect the genetic component in the covariance to be higher than the genetic components in the variances of the separate measurements.

Thus, longitudinal data bear more information about the underlying causes of phenotypic variability, because if the relative contribution of shared environment decreases when looking at correlations across time, this is an indication that not all shared environmental influences are influences that pertain to the sharing of environment during childhood, but are related to influences shared during the time of testing. The same applies to unique environmental influences: if these do not contribute to stability over time, then they are related to influences that individuals experienced during the time of testing or the time relatively recent to the time of testing, measurement error, or both.

Based on the above-mentioned findings and reasoning we expect relatively high heritabilities for vocabulary scores. We expect the shared environment component to be relatively small, or even 0 because vocabulary knowledge is highly related to intelligence which generally does not show influences from shared environmental factors in adults (Plomin et al., 2001; Rijsdijk et al., 2002). A recent review shows that shared environmental influences on IQ are already negligible starting from the age of 12 (Posthuma et al., 2002b). In addition to high heritabilities, we expect to find that the correlation across time is even more influenced by genetic factors.

Other longitudinal data on general cognitive ability in adults have indeed shown that the stability observed over time was largely due to genetic factors (Plomin et al., 1994). In contrast to the Plomin et al. study that included mostly older participants, our sample, in addition to an older cohort, includes a cohort of participants mostly in their 20s. The question that we can now address is whether the heritability of vocabulary, which can be regarded as an indicator of general cognitive ability, is stable across early and later adulthood.

\section{$\overline{\text { Method }}$ \\ Participants}

Nine hundred and ninety-seven members from 413 families with adult twins, registered in the Netherlands Twin Registry (Boomsma, 1998), participated in this study. The sample included 186 families with monozygotic (MZ) twins and 227 families with dizygotic (DZ) twins. At the first test occasion (t1) only twins were tested, comprising $94 \mathrm{MZ}$ twins and 1 MZ triplet, from a total number of 213 families. At t1 all had an age between 34 and 63 years $(M=44)$.

At the second test occasion (t2) 316 families participated, comprising 144 with MZ twins and 172 with DZ twins. This sample consisted of two distinguishable cohorts: one younger cohort where twins had an age between 18 and 34 ( $M=26$; $69 \mathrm{MZ}$ twin families and $91 \mathrm{DZ}$ twin families), and one older cohort where twins had an age between 36 and 71 years $(M=49$; 
$75 \mathrm{MZ}$ twin families and $81 \mathrm{DZ}$ twin families). At $\mathrm{t}$, siblings of twins were also included in the study. Siblings participating in the study were on average 0.5 years older than the twins ( $S D=5.3$ years).

Two hundred and seventeen individuals were tested both at $\mathrm{t} 1$ and $\mathrm{t} 2$, all twins and all from the older cohort: $52 \mathrm{MZ}$ and $63 \mathrm{DZ}$ twin pairs with some missing data.

There were differences in educational background between the two cohorts. In the older cohort tested at $\mathrm{t} 1,27 \%$ of the participants had completed university or a school for higher vocational training. This percentage was comparable with the percentage in the sample from the older cohort tested at t $2(30 \%)$. In contrast, $39 \%$ of the participants from the younger cohort, tested at $\mathrm{t} 2$, had completed university or a school for higher vocational training.

\section{Vocabulary}

Participants were given the vocabulary subtest from the Groninger Intelligentie Test (GIT; Snijders et al., 1983). At t1, this test was given as part of a study into the genetics of coronary heart disease (Snieder, 1996). At $t 2$, the test was part of an ongoing study on the genetics of adult brain function (Posthuma et al., 2002a; Posthuma et al., 2000). For each of 30 target words participants had to choose, from a list of four, the word that closely resembled the target word in meaning. A person's score was the total number of correctly chosen synonyms. These raw, non-standardized vocabulary scores were used in the analyses with the effects of age and sex explicitly modelled.

At the second test occasion both the Dutch version of the WAIS-III and the GIT vocabulary subtest were administered. The correlation between the GIT vocabulary score and the WAIS-III vocabulary score was .61 for males $(N=345)$ and .66 for females $(N=$ $443)$, both corrected for age. Only the GIT scores are analyzed here.

\section{Statistical Analyses}

Saturated models were fitted prior to the variance decomposition models. In the saturated models effects of sex, zygosity, and twin-singleton status on the variance and the means were tested, as well as effects of sex and twin-singleton status on the covariances. All data were used, including data from the triplets in the sample.

The vocabulary scores from the genetically related participants were analyzed using structural equation modeling, partitioning the variance into genetic and non-genetic components. As the sample consisted of families of different sizes, models were fitted to the raw data instead of covariance matrices. This was accomplished by using the rectangular data file option in $\mathrm{Mx}$ (Neale et al., 2003). When Mx experienced numerical difficulty in optimising the model fit, boundary constraints were imposed on the parameters.

The observed variances and covariances were decomposed into additive genetic $(\mathrm{A})$ and shared $(\mathrm{C})$ and non-shared (E) environmental components using maximum likelihood estimation. In the models, the correlation between the genetic latent variables was set to 1 for $\mathrm{MZ}$ twins, and to $1 / 2$ for $\mathrm{DZ}$ twins and non-twin siblings. Environmental factors incorporate those influences in the environment that are common to all siblings from one family $(\mathrm{C})$ and those influences that are not (E; Neale \& Cardon, 1992). Therefore, the correlations between the shared environmental factors were fixed at 1 for all members from the same family and the correlation between the non-shared environmental factors at 0 .

The data obtained at $\mathrm{t} 1$ and $\mathrm{t} 2$ were first analyzed separately to estimate the extent to which vocabulary scores are influenced by genetic factors and by shared environmental factors. Next, they were analyzed together in a bivariate analysis in order to study the stability of vocabulary over time. As we used the raw scores from the test, the effects of age and sex on the means were explicitly modelled in all analyses. As cohort effects (such as related to the number of years of formal education) exist for indices of crystallized/ verbal intelligence (Kaufman, 2001), cohort effects on parameter estimates were tested, as were sex effects.

All of the statistical tests reported here consist of comparing restricted models to models that are less restricted. Comparing the minus two times log-likelihood (-2LL) fit function of a model to the fit function of a less restricted model results in a statistic that is asymptotically $\chi^{2}$ distributed with degrees of freedom equal to the absolute difference of the degrees of freedom of both models. All statistical tests for significance were performed at an alpha level of $5 \%$.

\section{Results}

\section{Analysis of Vocabulary Scores Obtained at t1}

First, a saturated model was fitted that allowed means, variances, and covariances to be freely estimated for each of the five sex/zygosity groups (see Table 1). All data were used, including data from the triplets in the sample. Means were initially allowed to be different for MZ, DZ, and DOS pairs and were corrected for age and sex effects. Testing a number of hierarchically nested models revealed that the means were not dependent on zygosity status of the twins. Variances were similar for males and females and did not depend on zygosity status. Furthermore, MZ correlations were the same for males and females, and DZ correlations were the same for male-male twins, female-female twins, and opposite-sex twins. The final model, which did not fit significantly worse than the saturated model (see Table 1), showed an estimate of 15.5 for the mean of females and 16.3 for males, a significant difference, $\Delta-2 \mathrm{LL}=6.96, \Delta d f=1$. The variance estimate was 7.8 and the $\mathrm{MZ}$ and DZ correlation estimates were .58 and .41 respectively. The estimate for the effect of age on vocabulary score was -0.04 score point per increasing year, which was not significantly different from $0, \Delta-2 \mathrm{LL}=3.02, \Delta d f=1$. 
Table 1

Test Statistics for Equality of Means, Variances and Covariances at t1

\begin{tabular}{|c|c|c|c|c|c|}
\hline Model & \# Parameters & $-2 \mathrm{LL}$ & $d f$ & $\Delta-2 \mathrm{LL}$ & $\Delta d f$ \\
\hline $\begin{array}{l}\text { Saturated model: means (mz, dz, and dos tv } \\
\text { with sex and age covariates, } \\
\text { variance (mzm, dzm, mzf, dzf, dosm, dosf), } \\
\text { covariance (mzm, dzm, mzf, dzf, dos) }\end{array}$ & 16 & 2019.59 & 410 & & \\
\hline $\begin{array}{l}\text { Constraints: Equal means for all groups; } \\
\text { Equal vars for all groups } \\
M Z \text { and } D Z \text { covariances equal for sexes: } \\
m z M=m z f, d z M=d z f=\text { dos }\end{array}$ & 6 & 2024.70 & 420 & 5.11 & 10 \\
\hline
\end{tabular}

Based on this final model, the variance of vocabulary scores was decomposed into genetic and environmental components using an ACE model. The raw data log-likelihood fit index of the ACE model is displayed in Table 2, along with fit indices that indicate significant differences in fit between hierarchically nested models. The ACE model estimated relative contributions of $34 \%$ (95\% CI: 0-67), 24 $(0-53)$, and $42(31-57)$ for additive genetic, shared environmental, and non-shared environmental influences on vocabulary scores, respectively. Given these confidence intervals, the additive genetic and shared environment components are each not significantly different from 0 . However, as can be seen from the comparatively poor fit of the E model, there is significant familial resemblance. The statistical power seems insufficient to decompose this resemblance reliably into genetic and environmental components.

\section{Analyses of Vocabulary Scores Obtained at $\mathbf{t}$}

At $\mathrm{t} 2$, verbal intelligence scores were collected from twins and their siblings, a total of 788 individuals, of which 217 individuals (all twins) had been tested earlier at t1. Participants came from two age cohorts. Saturated models were fitted first for the two cohorts separately, after which the estimates were compared across cohorts. It was assumed that a correlation between two siblings was equal to a correlation between one sibling and a twin. A DZ twin-twin correlation was allowed to be different, however, as it can be expected that twins are more alike than siblings who do not share the same age. The means were corrected for age and sex effects.
The model fit indices for the younger cohort are displayed in Table 3. The means were not dependent on the zygosity status of the twins, nor did they differ between twins and siblings. Variances did not depend on zygosity status and were similar for twins and siblings, and for males and females. Furthermore, MZ covariances were the same for males and females, and DZ covariances were the same for male-male twins, female-female twins, and opposite-sex twins. The final model estimated a variance of 5.9. The DZ twin-twin correlation estimate was .28 , and only .12 for the sib-twin/sib-sib correlation. The estimate for the mean score was 10.6 for females and 11.0 for males, a nonsignificant difference, $\Delta-2 \mathrm{LL}=2.55$, $\Delta d f=1$. The effect of age was an increase of about 0.11 score points per increasing year, which was significantly different from $0, \Delta-2 \mathrm{LL}=11.26, \Delta d f=1$.

The same procedure was carried out for the older cohort (see Table 4). Again, the means were not dependent on the zygosity status of the twins, nor did they differ between twins and siblings. Variances did not depend on zygosity status and were similar for twins and siblings, and for males and females. Furthermore, MZ covariances were the same for males and females, and DZ covariances were the same for male-male twins, female-female twins, and opposite-sex twins. The final model estimated a variance of 6.4 and an MZ correlation of .63. The DZ twin-twin correlation estimate was .38 , and .23 for the sib-twin/sib-sib correlation. The estimate for the mean score was 14.9 for females and 15.8 for males, a significant difference $\Delta-2 \mathrm{LL}=9.38, \Delta d f=1$. The

Table 2

Test Statistics for the Significance of Variance Components at t1

\begin{tabular}{lccccc}
\hline Model & \# Parameters & $-2 \mathrm{LL}$ & $d f$ & $\Delta-2 \mathrm{LL}$ & $\Delta d f$ \\
\hline ACE model & 6 & 2024.70 & 420 & 5.11 & 3.20 \\
CE model & 5 & 2027.90 & 421 & 421 & 1 \\
AE model & 5 & 2026.83 & 422 & 57.41 & 1 \\
E model & 4 & 2082.11 & 2 \\
\hline
\end{tabular}

Note: Submodels $C E, A E$, and $E$ are compared with ACE model, which in turn is compared with the saturated model. The mean is corrected for sex and age effects. 
Table 3

Testing Equality of Means, Variances and Covariances in the Younger Cohort at t2

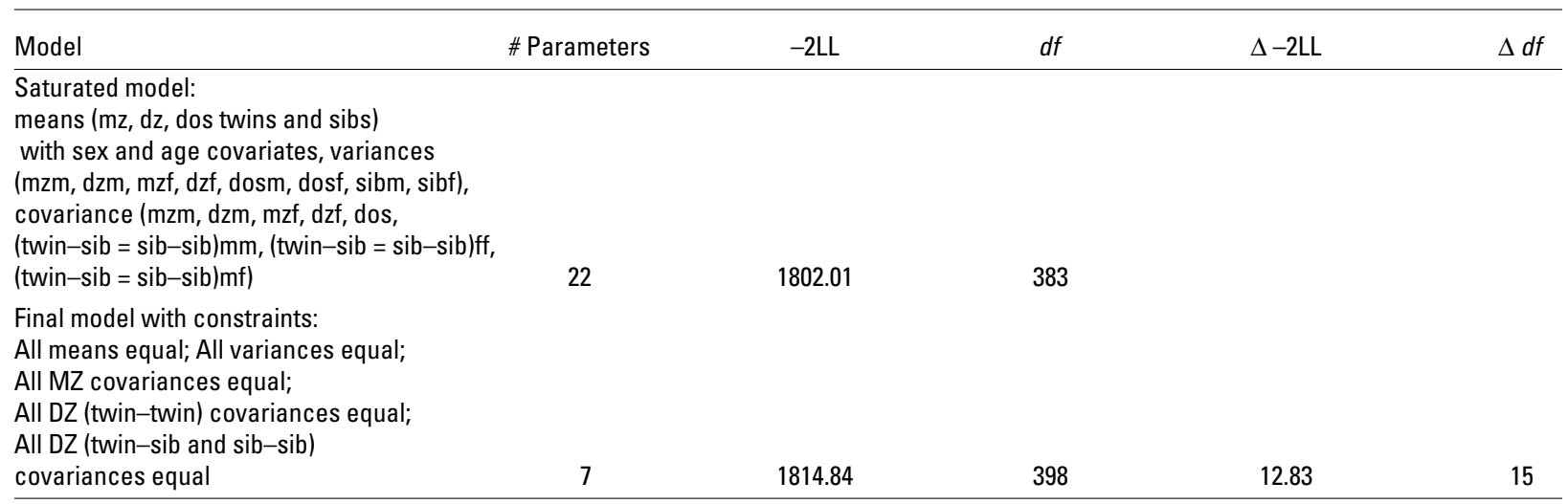

Note: Only the saturated and the final model are displayed. All intermediate nested models showed nonsignificant deteriorations in fit.

estimate for the effect of age was a decrease of about 0.02 score points per increasing year, but was not significantly different from $0, \Delta-2 \mathrm{LL}=0.84, \Delta d f=1$.

Next we tested whether these parameter estimates were equal across cohorts. The only parameter that was significantly different across cohorts was the parameter for the effect of the age covariate (see Table 5). In the final model, therefore, both the effect of the age covariate and the general mean were free to differ across cohorts. All other parameters could be equated without any significant loss of fit. The variance estimate was 6.2. Mean female score was estimated at 10.4 for the younger cohort and 14.9 in the older cohort, a significant cohort effect, $\Delta-2 \mathrm{LL}=11.22$, $\Delta d f=1$. Male scores were estimated to be generally 0.6 score points higher than female scores, also a significant difference, $\Delta-2 \mathrm{LL}=11.07, \Delta d f=1$. The estimates for the effects of the age covariates were the same as in the separate cohort analyses. The MZ correlation estimate was .67 (95\% CI: .57-.74). The estimates for the DZ twin-twin correlation and the twin-sib/sib-sib correlations were .34 (.18-.47) and .18 (.07-.29) respectively. These turned out not to be significantly different $(\Delta-2 \mathrm{LL}=3.15, \Delta d f=1)$. The general DZ correlation estimate was .23 (.13-.32). Therefore, for the variance decomposition, an ACE model was fitted, where A stands for additive genetic variance, $\mathrm{C}$ for variance shared by all family members regardless of being a twin or singleton, and $\mathrm{E}$ for variance that is unique for each family member.

The estimate for the additive genetic component was $63 \%$ (95\% CI: 51-72\%), for the shared environment component $0 \%(0-7 \%)$ and $37 \%(28-47 \%)$ for the unique environment component. These results suggest that resemblance in vocabulary scores among family members can be attributed entirely to their sharing of genes.

\section{Multivariate Analyses of All Data Available from t1 and t2}

The combined data from the two test occasions were analyzed in line with the findings from the two separate analyses. This means that an ACE model with Cholesky decomposition was fitted using covariates for age and sex. In line with the previous results, no sex or cohort effects were modeled apart from effects on the means. In line with the results from the t2

\section{Table 4}

Testing Equality of Means, Variances, and Covariances in the Older Cohort at t2

\begin{tabular}{|c|c|c|c|c|c|}
\hline Model & \# Parameters & $-2 \mathrm{LL}$ & $d f$ & $\Delta-2 \mathrm{LL}$ & $\Delta d f$ \\
\hline $\begin{array}{l}\text { Saturated model: } \\
\text { means (mz, dz, dos twins and sibs), } \\
\text { with sex and age covariates, variances } \\
\text { (mzm, dzm, mzf, dzf, dosm, dosf, sibm, sibf), } \\
\text { covariance (mzm, dzm, mzf, dzf, dos, } \\
\text { (twin-sib = sib-sib)mm, } \\
\text { (twin-sib = sib-sib)ff, (twin-sib = sib-sib)mf) }\end{array}$ & 22 & 1735.07 & 361 & & \\
\hline $\begin{array}{l}\text { Final model with constraints: } \\
\text { All means equal; All variances equal; } \\
\text { All MZ covariances equal; All DZ (twin-twin) } \\
\text { covariances equal; All DZ (twin-sib and } \\
\text { sib-sib) covariances equal }\end{array}$ & 7 & 1745.88 & 376 & 10.81 & 15 \\
\hline
\end{tabular}

Note: Only the saturated and the final model are displayed. All intermediate nested models showed nonsignificant deteriorations in fit. 
Table 5

Test Statistics for the Equality of Parameter Estimates Across the Two Age Cohorts at t2

\begin{tabular}{|c|c|c|c|c|}
\hline Model & $-2 \mathrm{LL}$ & \# parameters & $\Delta-2 \mathrm{LL}$ & $\Delta d f$ \\
\hline Saturated & 3560.72 & 14 & & \\
\hline Age covariates equal & 3571.65 & 13 & $10.93^{*}$ & 1 \\
\hline $\begin{array}{l}\text { Final model with equal variance } \\
\text { and equal sex covariate effects }\end{array}$ & 3565.46 & 9 & 4.74 & 5 \\
\hline
\end{tabular}

Note: Only the saturated, the final model, and one nested model are displayed. All intermediate nested models showed nonsignificant deteriorations in fit.

*Indicates a significant decrease in fit.

analysis, the sex covariate effect was equal for both cohorts at $\mathrm{t} 2$ but was free to differ from the effect at t1. In addition, also in line with the earlier results, the age covariate effect was allowed to differ for the two age cohorts at $\mathrm{t} 2$. This parameter for $\mathrm{t} 1 \mathrm{was}$ also free. Together with the nine ACE Cholesky parameters and three means, a total of 17 parameters were to be estimated, resulting in a -2LL of 5430.62 with 1197 degrees of freedom.

The previous analyses had shown that at $\mathrm{t} 2$, the effect of the sex covariate was equal across the two cohorts. As the difference in age between cohorts is larger than the difference in age across measurements (only 6 years), the effect of the sex covariate was restricted to be equal across both cohorts and measurements. This restricted model did not fit significantly worse, $\Delta-2 \mathrm{LL}=5430.67-5430.62=$ $0.05, \Delta d f=1$, and therefore was used as the basis for the parameter estimates displayed in Figure 1. From these parameters it can be inferred that the estimate for the genetic correlation is 1.00 (95\% CI: .90-1.00), meaning that all variance at both time points due to genetic variability comes from only one set of genes.
Also, at the second measurement no new shared environmental factors seem to play a role that were not already playing a part at the first testing occasion. In fact, the estimate for the shared environment component at t2 was .01 in this model (95\% CI: .00-.10). Due to this practically 0 variance, $\mathrm{Mx}$ estimated the shared environmental correlation at 1.00 with the confidence interval $-1.00-1.00$. There only seem to be new influences at $\mathrm{t} 2$ arising from environmental factors that are unique for each individual, including measurement error. The non-shared environmental correlation was .40 (95\% CI: .20-.56).

Following the tracing rules of path analysis, it can be calculated that the test-retest correlation was .74 and that $76 \%$ of this covariance between the two scores can be explained by genetic factors. Only $4 \%$ can be explained by shared environmental influences and the remaining $20 \%$ by non-shared environmental influences.

The parameter for the sex effect on the means was 0.62 , indicating that on average males score about half a point higher than females, a significant difference, $\Delta-2 \mathrm{LL}=12.89, \Delta d f=1$. The mean scores were

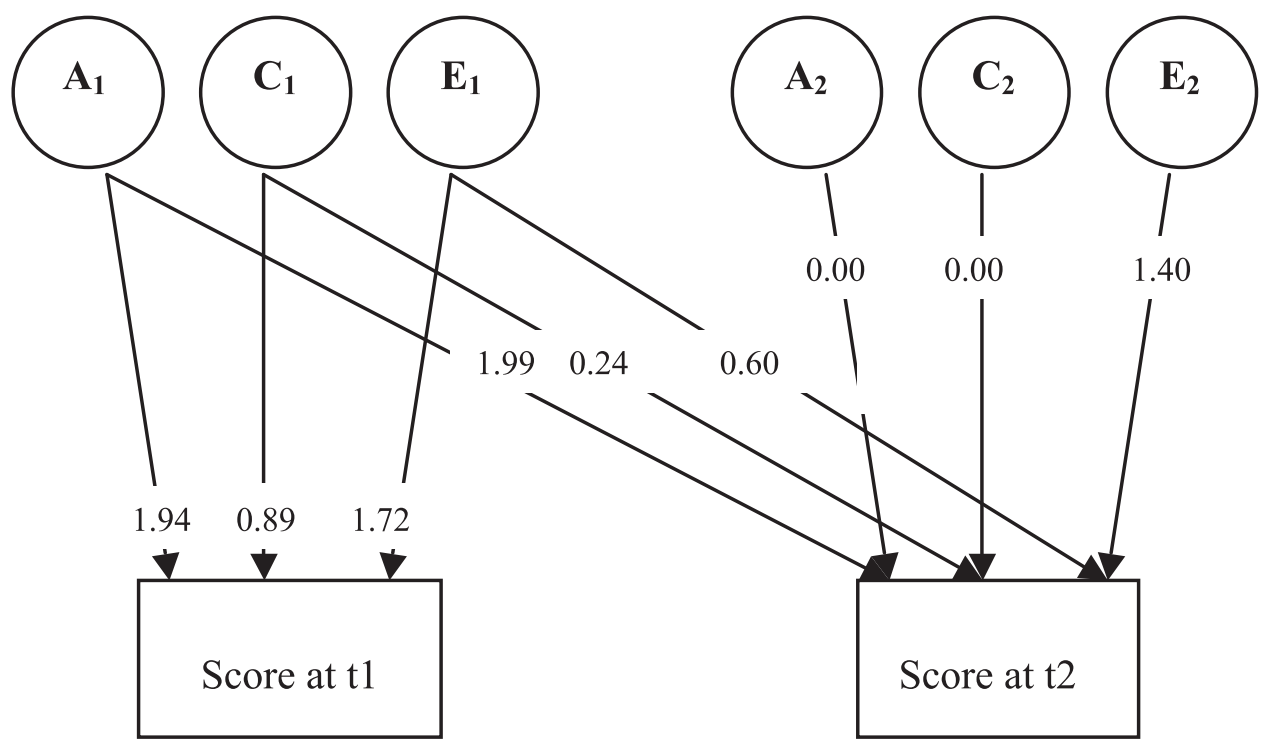

Figure 1

Cholesky decomposition of ACE factors and path coefficients. 
Table 6

Variance Components Estimates and 95\% Confidence Intervals for the Two Test Occasions (as Based on the Bivariate Analysis)

$\begin{array}{lcc}\text { Variance component in \% } & \text { t1 } & \text { t2 } \\ \text { Genetic } & 50(29-68) & 63(48-72) \\ \text { Shared environment } & 10(00-29) & 1(00-10) \\ \text { Non-shared environment } & 39(30-50) & 37(28-47)\end{array}$

higher for the older cohort (means 15.4 and 14.6 for $\mathrm{t} 1$ and $\mathrm{t} 2$ respectively) than for the younger cohort $(M$ $=10.1$ ). These means differed significantly, $\Delta-2 \mathrm{LL}=$ $16.46, \Delta d f=1$. The effect of age on vocabulary is also reflected by the size of the covariate effects: a significant 0.13 for the younger cohort, $\Delta-2 \mathrm{LL}=14.39$, $\Delta d f=1$, and nonsignificant -0.04 and -0.01 for the older cohort ( $\mathrm{t} 1$ and $\mathrm{t} 2$ respectively), $\Delta-2 \mathrm{LL}=3.35$, $\Delta d f=2$. These estimates suggest a nonlinear relationship between age and vocabulary, with an increase of the general mean in early adulthood and no change in later adulthood. Even though the older cohort had received less education than the younger cohort, they showed more vocabulary knowledge than the younger cohort.

\section{$\overline{\text { Discussion }}$}

Vocabulary knowledge was assessed in twins and their siblings, with a significant portion of the twins tested twice around 6 years apart. Heritability of vocabulary size was estimated, and an assessment was made of the underlying structure of the stability of scores over time. Test-retest correlation was .74 . Heritability was similar for males and females and for younger and older adults, and estimated between $50 \%$ at the first test occasion (only older adult twins) and $63 \%$ at the second test occasion (both younger and older adults, including siblings). Heritability estimates are equal across cohorts and are similar to those reported in adolescents (Bratko, 1996). It was found that the heritability of the two measures 6 years apart was due to only one set of genes. The covariance between the two measures could for the larger part $(76 \%)$ be explained by one genetic factor.

The shared environment does not seem to play any part when it comes to vocabulary knowledge. The nonsignificant shared environment variance component seems to imply that being brought up in one particular family or neighbourhood has no influence on knowing the meaning of less common words. However, it should be borne in mind that with the present design it was not possible to estimate genotype-environment correlation or genotype $\times$ environment interaction effects. As far as these effects may be related to shared environment, they are here part of the genetic component (Eaves et al., 1977; Lynch \& Walsh, 1998). It is also shown that time of testing does not affect mean score differences observed between families. The test therefore seems to be immune from temporary influences such as this month's talk of the town.

As for non-shared environmental influences, there are both time-specific influences (which of course include measurement error) and influences that were the same over time. We can therefore conclude that non-shared environmental influences are not all related to measurement error. In addition, we can conclude that not all person-specific effects result from a person being tested at a specific time, again an indication of the robustness of the test against temporary influences. It also means that the environment does influence vocabulary knowledge but that such influences are not shared between people from the same family. Stable person-specific influences might be thought of as the particular newspaper people read, what programs they watch on television, or interests in general.

The heritabilities at the specific time points of 50 and $63 \%$ are in fact estimates of heritabilities of observed scores, while $76 \%$ of the covariance explained by genetic factors can be regarded as an estimate of the heritability of true scores. This is because observed variances always include some measurement error, but variance that two measurements have in common presumably does not.

In conclusion, whether a person knows the meaning of "dehiscence" does not only depend on the environment he or she is brought up in. Of course, the language you speak and understand depends on the language spoken to you by your parents (Chomsky, 1957). However, the size and quality of people's vocabulary is not only dependent on the environment they are brought up in but can to a large extent be explained by genetic differences.

\section{Acknowledgment}

This study has been supported by grants from The Netherlands Organisation for Scientific Research, NWO nr. 051.02.060, 668.772, the Vrije Universiteit Universitair Stimuleringsfonds, VU-USF 96/22, and from the Human Frontiers of Science Program, RG0154/1998-B.

\section{References}

Aitken Harris, J. (in press). Measured intelligence, achievement, openness to experience, and creativity. Personality and Individual Differences.

Ashton, M. C., Lee, K., Vernon, P. A., \& Jang, K. L. (2000). Fluid intelligence, crystallized intelligence, and the openness/intellect factor. Journal of Research in Personality, 34(2), 198-207.

Boomsma, D. I. (1998). Twin registers in Europe: An overview. Twin Research, 1(1), 34-51.

Bratko, D. (1996). Twin study of verbal and spatial abilities. Personality and Individual Differences, 21(4), 621-624. 
Cattell, R. B. (1987). Intelligence: Its structure, growth and action. (Rev. ed.). Amsterdam: North-Holland.

Chomsky, N. (1957). Syntactic structures. The Hague, The Netherlands: Mouton.

Costa, J., \& McCrae, R. R. (1988). From catalog to classification: Murray's Needs and the Five-Factor Model. Journal of Personality and Social Psychology, 55(2), 258-265.

Dionne, G., Tremblay, R., Boivin, M., Laplante, D., \& Perusse, D. (2003). Physical aggression and expressive vocabulary in 19-month-old twins. Developmental Psychology, 39(2), 261-273.

Eaves, L. J., Last, K., Martin, N. G., \& Jinks, J. L. (1977). A progressive approach to non-additivity and genotype-environmental covariance in the analysis of human differences. The British Journal of Mathematical and Statistical Psychology, 30, 1-42.

Kaufman, A. S. (2001). WAIS-III IQs, Horn's theory, and generational changes from young adulthood to old age. Intelligence, 29(2), 131-167.

Luciano, M., Wright, M. J., Geffen, G. M., Geffen, L. B., Smith, G. A., Evans, D. M., et al. (2003). A genetic two-factor model of the covariation among a subset of Multidimensional Aptitude Battery and Wechsler Adult Intelligence Scale - Revised subtests. Intelligence, 31(6), 589-605.

Lynch, M., \& Walsh, B. (1998). Genetics and analysis of quantitative traits. Sunderland, MA: Sinauer.

Neale, M. C., Boker, S. M., Xie, G., \& Maes, H. H. (2003). Mx: Statistical modeling (6th ed.). Richmond, VA: Department of Psychiatry, VCU.

Neale, M. C., \& Cardon, L. R. (1992). Methodology for genetic studies of twins and families. Dordrecht, the Netherlands: Kluwer Academic Publishers.

Pedersen, N. L., Plomin, R., Nesselroade, J. R., \& McClearn, G. E. (1992). A quantitative genetic analysis of cognitive abilities during the second half of the life span. Psychological Science, 3(6), 346-353.

Plomin, R., DeFries, J. C., \& Loehlin, J.C. (1977). Genotype-environment interaction and correlation in the analysis of human behavior. Psychological Bulletin, 84, 309-322.

Plomin, R., DeFries, J. C., McClearn, G. E., \& McGuffin, P. (2001). Behavioral genetics (4th ed.). New York: Worth.

Plomin, R., Pedersen, N., Lichtenstein, P., \& McClearn, G. E. (1994). Variability and stability in cognitive abilities are largely genetic later in life. Behavior Genetics, 24(3), 207-215.

Posthuma, D., De Geus, E. J. C., Baaré, W. F. C., Hulshoff Poll, H. E., Kahn, R. S., \& Boomsma, D. I. (2002a). The association between brain volume and intelligence is of genetic origin. Nature Neuroscience, 5(2), 83-84.

Posthuma, D., De Geus, E. J. C., \& Boomsma, D. I. (2002b). Genetic contributions to anatomical, behavioral, and neurophysiological indices of cognition. In R. Plomin, J. C. DeFries, I. C. Craid, \& P. McGuffin (Eds.), Behavioral genetics in the postgenomic era. Washington, DC: APA Books.

Posthuma, D., De Geus, E. J. C., Neale, M. C., Hulshoff Pol, H. E., Baaré, W. F. C., Kahn, R. S., et al. (2000). Multivariate genetic analysis of brain structure in an extended twin design. Behavior Genetics, 30, 311-319.

Rijsdijk, F. V., Vernon, P. A., \& Boomsma, D. I. (2002). Application of hierarchical genetic models to Raven and WAIS subtests: A Dutch twin study. Behavior Genetics, 32(3), 199-210.

Rowe, D. C., Jacobson, K. C., \& Van den Oord, E. J. C. G. (1999). Genetic and environmental influences on vocabulary IQ: Parental education level as moderator. Child Development, 70(5), 1151-1162.

Scarr, S., \& McCartney, K. (1983). How people make their own environments: A theory of genotype $\times$ environment effects. Child Development, 54(2), 424-435.

Snieder, H. (1996). Genetic epidemiology of risk factors for coronary heart disease. Unpublished doctoral dissertation, Vrije Universiteit, Amsterdam, the Netherlands.

Snijders, J. T., Luteijn, F., \& Verhage, F. (1983). Groninger Intelligentie Test (GIT). Lisse, the Netherlands: Swets Test Publishers. 